PROCEEDINGS OF THE

AMERICAN MATHEMATICAL SOCIETY

Volume 127, Number 1, January 1999, Pages 199-203

S 0002-9939(99)04504-9

\title{
NONSTANDARD CHARACTERIZATION OF CONVERGENCE IN LAW FOR $D[0,1]$-VALUED RANDOM VARIABLES
}

\author{
D. LANDERS AND L. ROGGE
}

(Communicated by Stanley Sawyer)

\begin{abstract}
We prove for random variables with values in the space $D[0,1]$ of cadlag functions - endowed with the supremum metric - that convergence in law is equivalent to nonstandard constructions of internal $S$-cadlag processes, which represent up to an infinitesimal error the limit process. It is not required that the limit process is concentrated on the space $C[0,1]$, so that the theory is applicable to a wider class of limit processes as e.g. to Poisson processes or Gaussian processes. If we consider in $D[0,1]$ the Skorokhod metric instead of the supremum metric - we obtain a corresponding equivalence to constructions of internal processes with $S$-separated jumps. We apply these results to functional central limit theorems.
\end{abstract}

\section{INTRODUCTION}

In [1], [12], and [13] special processes such as Brownian motion, Levy's Brownian motion, and Brownian bridge were constructed by using nonstandard methods. In [6] it was shown that all these nonstandard constructions can be derived from a general result by means of suitable invariance principles. An essential assumption in [6] is that the considered processes a.e. have continuous paths. Therefore, many important processes, as for example Poisson processes or some Gaussian processes, cannot be dealt with using these methods. It is the purpose of this paper to close this gap.

In order to apply nonstandard methods, we work in this paper with a polysaturated nonstandard model.

Let $D[0,1]$ be the system of all functions $f:[0,1] \rightarrow \mathbb{R}$ which are continuous from the right and have left hand limits. These functions are called cadlag functions. Denote by ${ }^{*} D[0,1]$ the nonstandard extension of $D[0,1]$. We call a function $g \in$ ${ }^{*} D[0,1]$ an $S$-cadlag function if for every standard $r$ and every standard $\varepsilon>0$ there exists a standard $\delta>0$, such that

$$
\begin{gathered}
r<s \leq r+\delta \quad \Rightarrow \quad|g(s)-g(r)| \leq \varepsilon, \\
r-\delta \leq s, t<r \quad \Rightarrow \quad|g(s)-g(t)| \leq \varepsilon .
\end{gathered}
$$

If $s \in{ }^{*} \mathbb{R}$ is finite, we denote by ${ }^{\circ} s$ the standard part of $s$. We call $g \in{ }^{*} D[0,1]$ finite if $g(s)$ is finite for each $s \in{ }^{*}[0,1]$. If $g$ is finite, we write

${ }^{\circ} g$ for the function $[0,1] \ni t \rightarrow{ }^{\circ} g(t) \in \mathbb{R}$.

Received by the editors February 3, 1997 and, in revised form, May 8, 1997.

1991 Mathematics Subject Classification. Primary 28E05; Secondary 60B12.

Key words and phrases. Convergence in law for processes, nonstandard characterization. 
The finite $S$-cadlag functions $g \in{ }^{*} D[0,1]$ are the nearstandard-points of ${ }^{*} D[0,1]$, where $D[0,1]$ is endowed with the uniform metric $\delta_{\infty}$. The standard part of $g$, denoted by $s t_{\delta_{\infty}}(g)$, is given by ${ }^{\circ} g$ (see Lemma 2.4).

A function $g \in{ }^{*} D[0,1]$ has $S$-separated jumps if for every standard $\varepsilon>0$ there exist a standard $\delta>0$ and $0=r_{0}<r_{1}<\ldots<r_{m}=1$ with $r_{j}-r_{j-1}>\delta$ for $1 \leq j \leq m$ and $\left|g(s)-g\left(r_{j-1}\right)\right| \leq \varepsilon$ for $r_{j-1} \leq s<r_{j}$ and $1 \leq j \leq m$.

The finite $g \in{ }^{*} D[0,1]$ with $S$-separated jumps are the nearstandard-points of ${ }^{*} D[0,1]$ with respect to the Skorokhod metric $\delta_{S}$ on $D[0,1]$. The standard part of $g$, denoted by $s t_{\delta_{S}}(g)$, is given by ${ }^{\oplus} g$ (see Lemma 2.5 ), where ${ }^{\oplus} g:[0,1] \rightarrow \mathbb{R}$ is defined by ${ }^{\oplus} g(t)=\lim _{r \downarrow t}{ }^{\circ} g(r)$ for $t \in[0,1]$.

\section{THE RESUlts}

Let $(M, \delta)$ be a metric space with Borel $\sigma$-algebra $\mathcal{B}$. If $\left(\Omega_{n}, \mathcal{A}_{n}, P_{n}\right)$ are probability spaces and $Y_{n}: \Omega_{n} \rightarrow M$ are $\mathcal{A}_{n}, \mathcal{B}$-measurable random variables for $n \in \mathbb{N}$, we denote by $\left(\Omega_{h}, \mathcal{A}_{h}, P_{h}, Y_{h}\right)$ for $h \in{ }^{*} \mathbb{N} \backslash \mathbb{N}$ the nonstandard extension of the sequence $\mathbb{N} \ni n \rightarrow\left(\Omega_{n}, \mathcal{A}_{n}, P_{n}, Y_{n}\right)$ at the point $h$. Then $\left(\Omega_{h}, \mathcal{A}_{h}, P_{h}\right)$ is an internal probability space and $Y_{h}: \Omega_{h} \rightarrow{ }^{*} M$ is an internal function with $Y_{h}^{-1}(B) \in \mathcal{A}_{h}$ for $B \in{ }^{*} \mathcal{B}$.

The nonstandard constructions of processes considered in this paper use the concept of a Loeb measure, which was introduced in [7] and gave nonstandard measure theory and probability theory a fresh impetus. We denote the Loeb measure derived from $P_{h}$ by $P_{h}^{L}$.

The following theorem is known if $\left(\Omega_{n}, \mathcal{A}_{n}\right) \equiv(M, \mathcal{B})$ and $Y_{n} \equiv i d_{M}$ for all $n \in \mathbb{N}$, where $i d_{M}$ is the identity map on $M$ (see Anderson-Rashid [2] and Loeb [10]). Loeb himself used this special version of Theorem 2.1 and similar results in a series of fundamental papers on axiomatic potential theory and harmonic analysis (see Loeb [8], [9], [11]).

2.1 Theorem. Let $(M, \delta)$ be a metric space with Borel $\sigma$-algebra $\mathcal{B}$. Let $Y_{n}$ be $M$ valued random variables. Let $Q$ be a Radon p-measure on $\mathcal{B}$. Then the $Y_{n}$ converge in law to $Q$ if and only if for each $h \in{ }^{*} \mathbb{N} \backslash \mathbb{N}$ the following conditions are fulfilled:

1. $Y_{h} \in n s_{\delta}\left({ }^{*} M\right) P_{h}^{L}$-a.e.

2. $P_{h}^{L}\left\{\operatorname{st}_{\delta}\left(Y_{h}\right) \in B\right\}=Q(B)$ for all $B \in \mathcal{B}$.

Proof. Let $Q_{n}:=\left(P_{n}\right)_{Y_{n}}$ be the law of $Y_{n}$. Then the $Y_{n}$ converge in law to $Q$ if and only if

$$
Q_{h}=\left(P_{h}\right)_{Y_{h}} \underset{w}{\approx} Q \quad \text { for all } \quad h \in * \mathbb{N} \backslash \mathbb{N},
$$

where $\approx_{w}$ means infinitesimal-closeness with respect to the weak topology. According to a slight modification of Theorem 33.1 of [5], (1) is equivalent to

$$
Q(O) \leq Q_{h}^{L}\left({ }^{*} O\right) \text { for all open } O \subset M \text { and all } h \in{ }^{*} \mathbb{N} \backslash \mathbb{N} \text {. }
$$

As $Q$ is a Radon measure, it follows from Theorem 32.6 of [5] that (2) is equivalent to

$$
\begin{aligned}
& \text { a) } \quad Q_{h}^{L}\left(n s_{\delta}\left({ }^{*} M\right)\right)=1 \quad \text { for all } \quad h \in{ }^{*} \mathbb{N} \backslash \mathbb{N} \text {, } \\
& \text { b) } \quad Q=\left(Q_{h}^{L}\right)_{s t_{\delta}} \quad \text { for all } \quad h \in * \mathbb{N} \backslash \mathbb{N} \text {. }
\end{aligned}
$$

Finally, according to Lemma 2 of [6], (3) is equivalent to

$$
\begin{aligned}
& \text { a) } \quad Y_{h}(\omega) \in n s_{\delta}\left({ }^{*} M\right) \text { for } P_{h}^{L} \text { - a.a. } \omega \text { for all } h \in * \mathbb{N} \backslash \mathbb{N} \text {, } \\
& \text { b) } \quad Q=\left(P_{h}^{L}\right)_{s t_{\delta} \circ Y_{h}} \quad \text { for all } h \in{ }^{*} \mathbb{N} \backslash \mathbb{N} \text {. }
\end{aligned}
$$

This proves the assertion. 
2.2 Corollary. Let $D[0,1]$ be endowed with the uniform metric $\delta_{\infty}$. Let $Y_{n}$ be $D[0,1]$-valued random variables. Let $Q$ be a Radon p-measure on the Borel $\sigma$ algebra $\mathcal{B}_{\infty}$ of $\left(D[0,1], \delta_{\infty}\right)$. Then the $Y_{n}$ converge in law to $Q$ if and only if for each $h \in{ }^{*} \mathbb{N} \backslash \mathbb{N}$ the following conditions are fulfilled:

1. $Y_{h}$ is $P_{h}^{L}$-a.e. finite and $S$-cadlag.

2. $P_{h}^{L}\left\{{ }^{\circ} Y_{h} \in B\right\}=Q(B)$ for all $B \in \mathcal{B}_{\infty}$.

Proof. Apply Theorem 2.1 to $(M, \delta) \equiv\left(D[0,1], \delta_{\infty}\right)$ and use Lemma 2.4.

As $\left(D[0,1], \delta_{\infty}\right)$ is a complete metric space, a probability measure on $\mathcal{B}_{\infty}$ is a Radon measure if and only if it is $\tau$-smooth. All probability measures on $\mathcal{B}_{\infty}$ are $\tau$-smooth if and only if each discrete subset of $D[0,1]$ has nonmeasurable cardinal (see Theorem 2 of Billingsley [3], p. 235).

If we assume the continuum hypothesis, then $D[0,1]$ and hence all discrete subsets of $D[0,1]$ have nonmeasurable cardinals. Therefore, in this case, each probability measure on $\mathcal{B}_{\infty}$ is a Radon measure, so that the assumption on $Q$ in Corollary 2.2 is automatically fulfilled.

If we use instead of $\delta_{\infty}$ the Skorokhod metric $\delta_{S}$ on $D[0,1]$, then $\left(D[0,1], \delta_{S}\right)$ is a complete separable metric space (see $\S 14$ of Billingsley [3]), so that each measure on the Borel $\sigma$-algebra $\mathcal{B}_{S}$ is a Radon measure. Therefore we obtain

2.3 Corollary. Let $D[0,1]$ be endowed with the Skorokhod metric $\delta_{S}$. Let $Y_{n}$ be $D[0,1]$-valued random variables. Let $Q$ be a p-measure on the Borel $\sigma$-algebra $\mathcal{B}_{S}$ of $\left(D[0,1], \delta_{S}\right)$. Then the $Y_{n}$ converge in law to $Q$ if and only if for each $h \in{ }^{*} \mathbb{N} \backslash \mathbb{N}$ the following conditions are fulfilled:

1. $Y_{h}$ is $P_{h}^{L}$-a.e. finite with $S$-separated jumps.

2. $P_{h}^{L}\left\{{ }^{\oplus} Y_{h} \in B\right\}=Q(B)$ for all $B \in \mathcal{B}_{S}$.

Proof. Apply Theorem 2.1 to $(M, \delta) \equiv\left(D[0,1], \delta_{S}\right)$ and use Lemma 2.5.

2.4 Lemma. Let $D:=D[0,1]$ be endowed with the uniform metric $\delta_{\infty}$. Then $g \in n s_{\delta_{\infty}}\left({ }^{*} D\right)$ if and only if $g$ is finite and $S$-cadlag. If $g \in n s_{\delta_{\infty}}\left({ }^{*} D\right)$, then $s t_{\delta_{\infty}}(g)={ }^{\circ} g$.

Proof. Let $g \in n s_{\delta_{\infty}}\left({ }^{*} D\right)$, then there exists $f \in D$ with $g \approx_{\delta_{\infty}} f$. As $g(s) \approx{ }^{*} f(s)$ for all $s \in{ }^{*}[0,1], g(s)$ is finite for all $s \in{ }^{*}[0,1]$ and $s t_{\delta_{\infty}}(g)={ }^{\circ} g$. As ${ }^{*} f$ is $S$-cadlag and $g(s) \approx{ }^{*} f(s)$ for all $s \in{ }^{*}[0,1], g$ is $S$-cadlag, too.

For the converse, let $g \in{ }^{*} D$ be $S$-cadlag and finite. Put $f(t):={ }^{\circ} g(t)$ for $t \in[0,1]$. Then $f \in D$, and it suffices to show that

$$
\begin{aligned}
& s \in{ }^{*}[0,1] \wedge{ }^{\circ} s<s \Rightarrow g(s) \approx{ }^{*} f(s), \\
& s \in{ }^{*}[0,1] \wedge s<{ }^{\circ} s \Rightarrow g(s) \approx{ }^{*} f(s) .
\end{aligned}
$$

As $g$ and ${ }^{*} f$ are $S$-cadlag, (1) follows from $g(s) \approx g\left({ }^{\circ} s\right) \approx f\left({ }^{\circ} s\right) \approx{ }^{*} f(s)$.

To prove (2), let $s \in{ }^{*}[0,1]$ and $\varepsilon \in \mathbb{R}_{+}$be given. As $g$ and ${ }^{*} f$ are $S$-cadlag, there exists $\delta \in \mathbb{R}_{+}$with $\left|{ }^{*} f\left(s_{1}\right)-{ }^{*} f\left(s_{2}\right)\right| \leq \varepsilon$ and $\left|g\left(s_{1}\right)-g\left(s_{2}\right)\right| \leq \varepsilon$ for all $s_{1}, s_{2} \in{ }^{*}[0,1]$ with ${ }^{\circ} s-\delta \leq s_{1}, s_{2}<{ }^{\circ} s$. As $f\left({ }^{\circ} s-\delta\right) \approx g\left({ }^{\circ} s-\delta\right)$ we obtain $\left|g\left(s_{2}\right)-{ }^{*} f\left(s_{2}\right)\right| \leq 3 \varepsilon$ for all $s_{2} \in{ }^{*}[0,1]$ with ${ }^{\circ} s-\delta \leq s_{2}<{ }^{\circ} s$. This implies (2).

2.5 Lemma. Let $D:=D[0,1]$ be endowed with the Skorokhod metric $\delta_{S}$. Then $g \in n s_{\delta_{S}}\left({ }^{*} D\right)$ if and only if $g$ is finite and has $S$-separated jumps. If $g \in n s_{\delta_{S}}\left({ }^{*} D\right)$, then $s t_{\delta_{S}}(g)={ }^{\oplus} g$.

Proof. See 5.3.7 and 5.3.6 of Stroyan-Bayod [14]. 
Now we give applications of Corollaries 2.2 and 2.3 to special stochastic processes.

2.6 Application of 2.2. For each $n \in \mathbb{N}$ let $\xi_{n, 1}, \ldots, \xi_{n, n}$ be independent random variables with $P_{n}\left\{\xi_{n, k}=1\right\}=p_{n}$ and $P_{n}\left\{\xi_{n, k}=0\right\}=1-p_{n}$ for $k=1, \ldots, n$. Define $Y_{n}$ by $\left(Y_{n}(\omega)\right)(t):=\sum_{k \leq n t} \xi_{n, k}(\omega)$. Assume that $n p_{n} \rightarrow \lambda$. Then for each $h \in * \mathbb{N} \backslash \mathbb{N}$ we have:

1. $Y_{h}$ is $P_{h}^{L}$-a.e. finite and $S$-cadlag.

2. ${ }^{\circ} Y_{h}$ is a Poisson-process with parameter $\lambda$, where

$$
\circ\left(Y_{h}(\omega)\right)(t)=\circ\left(\sum_{k \leq h t} \xi_{h, k}(\omega)\right) .
$$

Proof. According to Billingsley [3, p. 143, Problem 3], the $Y_{n}$ converge in law to a measure $Q$ that is the law of a Poisson process with parameter $\lambda$. Hence the assertion follows from Corollary 2.2.

2.7 Application of 2.3. Let $\xi_{n}: \Omega \rightarrow[0,1], n \in \mathbb{N}$, be independent random variables with common distribution function $F$. Let

$$
\left(Y_{n}(\omega)\right)(t):=\sqrt{n}\left(F_{n}(t, \omega)-F(t)\right),
$$

where $F_{n}$ is the empirical distribution function of $\xi_{1}, \ldots, \xi_{n}$. Then for each $h \in$ $* \mathbb{N} \backslash \mathbb{N}$ we have:

1. $Y_{h}$ is $P^{L}$-a.e. finite with $S$-separated jumps.

2. ${ }^{\oplus} Y_{h}$ is a Gaussian process in $D$ with zero means, and $\operatorname{cov}\left({ }^{\oplus} Y_{h}(s),{ }^{\oplus} Y_{h}(t)\right)=$ $F(s)(1-F(t))$ for $0 \leq s \leq t \leq 1$.

Proof. According to Billingsley [3, p. 141, Theorem 16.4], $Y_{n}$ converges in law to a measure $Q$ on the Borel $\sigma$-algebra $\mathcal{B}_{S}$ that is the law of a Gaussian process in $D[0,1]$ with zero means and covariances $F(s)(1-F(t)), s \leq t$. Hence the assertion follows from Corollary 2.3.

\section{REFERENCES}

[1] Robert M. Anderson, A nonstandard representation for Brownian motion and Itô integration, Israel J. Math. 25 (1976), 15-46.

[2] Robert M. Anderson and Salim Rashid, A nonstandard characterization of weak convergence, Proc. Amer. Math. Soc. 69 (1978), 327-332. MR 58:1073

[3] Patrick Billingsley, Convergence of probability measures, Wiley, New York and Toronto, 1968. MR 38:1718

[4] Dieter Landers and Lothar Rogge, Universal Loeb-measurability of sets and of the standard part map with applications, Trans. Amer. Math. Soc. 304 (1987), 229-243. MR 89d:28015

[5] Dieter Landers and Lothar Rogge, Nichtstandard Analysis, Springer Verlag, Heidelberg and New York, 1994. MR 95i:03140

[6] Dieter Landers and Lothar Rogge, Nonstandard characterization for a general invariance principle, Advances in analysis, probability and mathematical physics (edited by Albeverio, Luxemburg, Wolff), Kluver Publ. Comp., 1995, pp. 176-185. MR 96i:28024

[7] Peter A. Loeb, Conversion from nonstandard to standard measure spaces and applications in probability theory, Trans. Amer. Math. Soc. 211 (1975), 113-122. MR 52:10980

[8] Peter A. Loeb, Applications of nonstandard analysis to ideal boundaries in potential theory, Israel J. Math. 25 (1976), 154-187. MR 56:15961

[9] Peter A. Loeb, A generalization of the Riesz-Herglotz theorem on representing measures, Proc. Amer. Math. Soc. 71 (1978), 65-68. MR 58:28574

[10] Peter A. Loeb, Weak limits of measures and the standard part map, Proc. Amer. Math. Soc. 77 (1979), 128-135. MR 80i:28020

[11] Peter A. Loeb, A construction of representing measures for elliptic and parabolic differential equations, Math. Ann. 260 (1982), 51-56. MR 83i:31015 
[12] G. R. Mendieta, Two hyperfinite constructions of the Brownian bridge, Stochastic Anal. Appl. 7 (1989), 75-88. MR 90e:60047

[13] A. Stoll, A nonstandard construction of the Levy Brownian motion, Probab. Theory Related Fields 71 (1986), 321-334. MR 87h:60149

[14] K.D. Stroyan and J.M. Bayod, Foundations of infinitesimal stochastic analysis, North-Holland, Amsterdam and New York, 1986. MR 87m:60001

Dieter Landers, Mathematisches Institut Der Universität Zu Köln, Weyertal 86, D50931 Köln, Germany

E-mail address: landers@mi.uni-koeln.de

Lothar Rogge, Fachbereich Mathematik der Gerhard-Mercator-Universität GhS Duisburg, Lotharstr. 65, D-47048 Duisburg, Germany

E-mail address: rogge@math.uni-duisburg.de 